EPJ Web of Conferences 38, 17010 (2012)

DOI: $10.1051 /$ epjconf/20123817010

(c) Owned by the authors, published by EDP Sciences, 2012

\title{
On the direct and semidirect neutron radiative capture by medium-heavy mass nuclei
}

\author{
B.A. Tulupov ${ }^{1, a}$ and M.H. Urin ${ }^{2}$ \\ 1 Institute for Nuclear Research, RAS, Moscow, Russia \\ 2 National Research Nuclear University "MEPhI", Moscow, Russia
}

\begin{abstract}
The new semimicroscopic model (particle-hole dispersive optical model) recently developed for describing high-energy particle-hole-type excitations in medium-heavy mass nuclei is applied for the description of the simplest photonuclear reactions accompanied by excitation of the isovector giant dipole and quadrupole resonances. The calculated reaction cross sections and their derivatives are compared with corresponding experimental data and also with the similar results obtained within the previously developed semimicroscopic approach to the description of the giant resonances.
\end{abstract}

\section{Introduction}

The simplest photonuclear reactions (photoabsorption, direct+semidirect (DSD) photonucleon and inverse reactions) many years were a powerful tool of experimental studies of the isovector giant dipole and quadrupole resonances (IVGDR and IVGQR, respectively) (see, e.g. [1]). Theoretical description of above mentioned reactions is most adequate within microscopic and semimicroscopic approaches, allowing take into account the main relaxation modes of giant resonances (GRs). In case of GRs in medium-heavy mass 'hard' spherical nuclei (in particular, in singly- and doubly-closed-shell nuclei) these modes are: (i) particlehole (p-h) strength distribution, or Landau damping, which is a result of shell structure of nuclei; (ii) coupling of the (p-h)-type states, forming a given GR, to the single-particle (s. p.) continuum, that leads to GR direct-nucleon decay, or to the DSD reactions with one nucleon in the continuum; (iii) coupling of the mentioned (p-h)-type states to manyquasiparticle configurations (chaotic states), that leads to the spreading effect. These relaxation modes are taken into account within the semimicroscopic approach to the description of GRs (SMAGR) based on the versions of the continuum-Random-Phase-Approximation (cRPA) [2,3]. Within this approach Landau damping and coupling to the s. p. continuum are considered microscopically, using the cRPA, while the spreading effect is described in a phenomenological way in terms of the energy-dependent imaginary part $W(\omega)$ of the effective s. p. optical-model potential directly used in the cRPA equations. In implementations of the SMAGR a phenomenological mean field and the Landau-Migdal (momentum-independent) p-h interaction connected by some self-consistency conditions are used, as the input quantities. The above mentioned imaginary part is parameterized by an universal function, having the saturation-like energy dependence, with intensity $\alpha\left(\mathrm{MeV}^{-1}\right)$ chosen to describe the experimental total width of a given GR. Being appeared due to the spreading effect, the real part of the effective s. p. optical-model po-

\footnotetext{
${ }^{a}$ e-mail: tulupov@cpc.inr.ac.ru
}

tential $P(\omega)$ is determined by the imaginary part via the proper dispersive relationship [4]. The unique feature of the SMAGR is its ability to describe direct-nucleon-decay properties of various GRs without the use of specific adjustable parameters. (A number of implementations of the approach are briefly reviewed in [2,3]). As applied to the description of photoabsorption within the above described approach, it was found the necessity to take the isovector momentum-dependent forces into account to reproduce in partially self-consistent cRPA calculations the observed IVGDR energy and exceeding the experimental integral photoabsorption cross section over the Thomas-ReichKuhn sum rule. To formulate the cRPA equations in a closed form, these forces are taken as separable p-h dipole-dipole and quadrupole-quadrupole isovector velocity-dependent interactions (with the dimensionless intensities $k_{L}^{\prime}, L=1,2$ ). This extended version of the SMAGR is used in our recent paper [5], where reasonable description of the photoabsorption and the partial differential DSD $(\gamma, n)-$ and $(n, \gamma)$-reaction cross sections is given for a number of medium-heavy mass spherical nuclei. In particular, the asymmetry (with respect to $90^{\circ}$ ) of the partial differential cross sections of the $(\gamma, n)$ - and $(n, \gamma)$ - reactions was also described. Being determined by interference of the $E 1$ - and E2-reaction amplitudes (the latter is due to the p-h interaction), this asymmetry exhibits a non-monotonic energy dependence in a vicinity of the IVGQR and is the most suitable object for the study of this resonance in photonuclear reactions.

Within the SMAGR phenomenological treatment of the spreading effect consists in the known substitution $\omega \rightarrow \omega+i W(\omega)-P(\omega)$ [5] in cRPA equations and, therefore, was expected to be valid in the 'pole' approximation (i.e., in the energy region of a given GR) [2,3]. In some cases it is necessary to know properties of (p-h)-type excitations at energies far from the corresponding GR energy. For example, the above discussed asymmetry is determined, in particular, by the E1-reaction amplitude taken in the energy region of the IVGQR [5]. Recently, a semimicroscopic model (the $\mathrm{p}-\mathrm{h}$ dispersive optical model (PHDOM)) is developed to describe properties of (p-h)-type 
excitations at arbitrary (but high enough) energies $[3,6]$. Within the PHDOM and SMAGR the same input quantities and model parameters are used. Moreover, in the energy region of a given GR the results obtained within both models are expected to be closely related. Differences might appear outside the GR region. In other words, the PHDOM is the verification and the extension of the SMAGR. In the 'pole' approximation the energy-averaged 'free' $\mathrm{p}$-h Green function is taken as:

$$
\bar{A}_{0}^{\text {pole }}(\omega)=A_{0}^{c R P A}(\omega \rightarrow \omega+i W(\omega)-P(\omega)) .
$$

The main new point in formulation of the PHDOM is the corresponding expression for the energy-averaged 'free' $\mathrm{p}$ $\mathrm{h}$ radial Green function $\bar{A}_{0}(\omega)$ which is different from that used within the SMAGR. The easiest way to see the distinction between these quantities is the use of $\lambda$-representation (' $\lambda$ ' is the total set of single - quasiparticle quantum numbers). In this case the quantity $A_{0}^{R P A}(\omega)$ is well known [7]:

$$
\left(A_{0}^{R P A}\right)_{\lambda \lambda^{\prime}}(\omega)=\frac{n_{\lambda}-n_{\lambda^{\prime}}}{\varepsilon_{\lambda}-\varepsilon_{\lambda^{\prime}}-\omega},
$$

whereas $\left(\bar{A}_{0}\right)_{\lambda \lambda^{\prime}}(\omega)$ has the form $[3,6]$ :

$$
\begin{aligned}
& \left(\bar{A}_{0}\right)_{\lambda \lambda^{\prime}}(\omega)= \\
& \quad=\frac{n_{\lambda}-n_{\lambda^{\prime}}}{\varepsilon_{\lambda}-\varepsilon_{\lambda^{\prime}}-\omega+\left(n_{\lambda}-n_{\lambda^{\prime}}\right)(i W(\omega)-P(\omega)) f_{\lambda} f_{\lambda^{\prime}}} .
\end{aligned}
$$

The meaning of the quantities introduced in (3) is determined in $[3,6]$. In the present work some calculation results obtained within the PHDOM for the photoabsorption and the partial differential $(n, \gamma)$-reaction cross sections are shown and compared with the results obtained within the SMAGR [5] and also with the corresponding experimental data.

\section{Calculation results}

Basic relationships for the description within the cRPA of the simplest photo-nuclear reactions, namely, the strength functions, the total photoabsorption cross sections, the partial differential cross sections of the $(n, \gamma)$-reaction, the anisotropy parameter $a_{2}$ and the asymmetry are given in [5]. Modifications of these relationships made for taking the spreading effect into account within the SMAGR and PHDOM are discussed above. Following [5], we employ in calculations the partially self-consistent phenomenological mean field, described in [8]. Due to the isovector selfconsistency condition, the (dimensionless) intensity $f^{\prime}$ of the isovector spinless part of the Landau-Migdal p-h interaction is found from description of the symmetry potential. Therefore, only two above-mentioned adjustable parameters $\alpha$ and $k_{1}^{\prime}$, determining, respectively, the total width and energy of the IVGDR, are used to reproduce in calculations within the SMAGR and PHDOM the experimental photoabsorption cross section. Quality of such description of the IVGDR one can see from figures 1-3, where the corresponding data are shown for ${ }^{208} \mathrm{~Pb},{ }^{140} \mathrm{Ce}$ and ${ }^{89} \mathrm{Y}$. The adjusted parameters are listed in Table 1. The partial differential DSD $(n, \gamma)$-reaction cross sections are calculated without use of the additional adjusted parameters. The calculated cross sections at $90^{\circ}$ are shown in figures 4-6 in

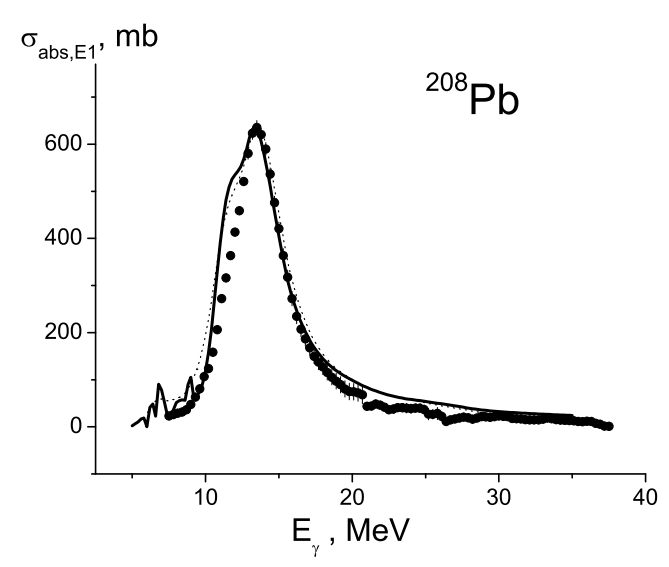

Fig. 1. The calculated $E 1$ total photoabsorption cross section for ${ }^{208} \mathrm{~Pb}$ in comparison with the experimental data [9].

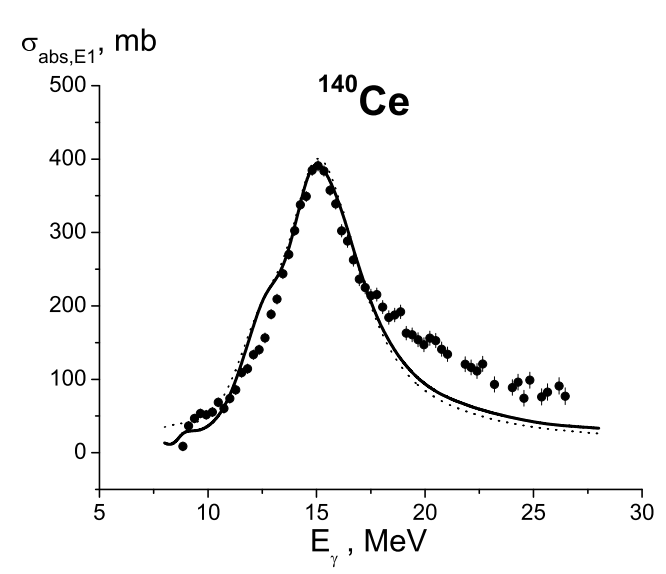

Fig. 2. The calculated $E 1$ total photoabsorption cross section for ${ }^{140} \mathrm{Ce}$ in comparison with the experimental data [9].

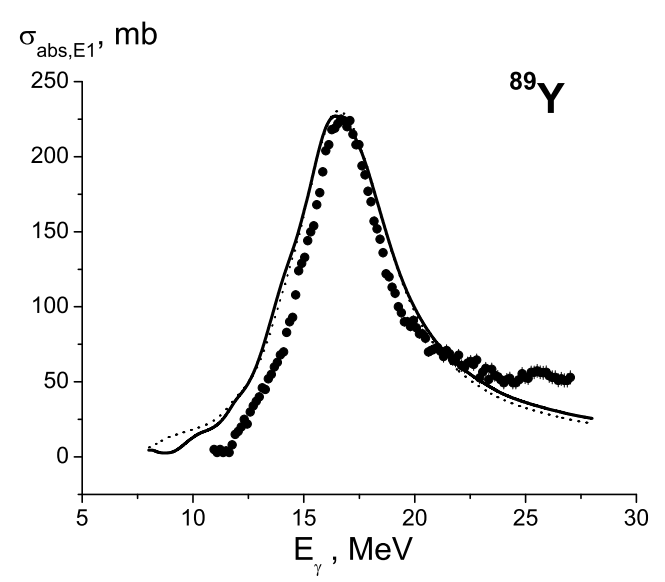

Fig. 3. The calculated $E 1$ total photoabsorption cross section for ${ }^{89} \mathrm{Y}$ in comparison with the experimental data [9]. 
Table 1. The adjustable parameters used in the studies of the simplest photonuclear reactions in the IVGDR energy region.

\begin{tabular}{|c|c|c|}
\hline Nucleus & $\begin{array}{c}\alpha, \mathrm{Mev}^{-1} \\
\text { SMAGR | PHDOM }\end{array}$ & SMAGR $\stackrel{k_{1}^{\prime}}{\text { | PHDOM }}$ \\
\hline${ }^{89} \mathrm{Y}$ & $0.125 \mid 0.13$ & $0.3 \mid 0.3$ \\
\hline${ }^{140} \mathrm{Ce}$ & $0.105 \mid 0.115$ & $0.3 \mid 0.3$ \\
\hline${ }^{208} \mathrm{~Pb}$ & $0.10 \mid 0.105$ & $0.4 \mid 0.35$ \\
\hline
\end{tabular}

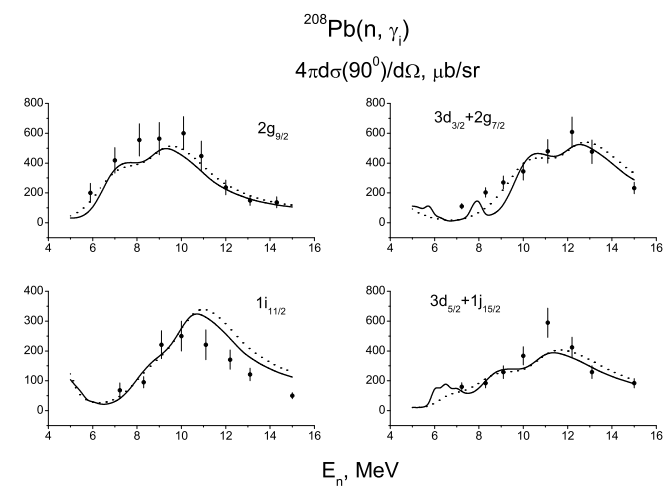

Fig. 4. The calculated partial differential cross sections of the $(n, \gamma)$-reaction for ${ }^{208} \mathrm{~Pb}$. The experimental data are taken from Ref. [10].

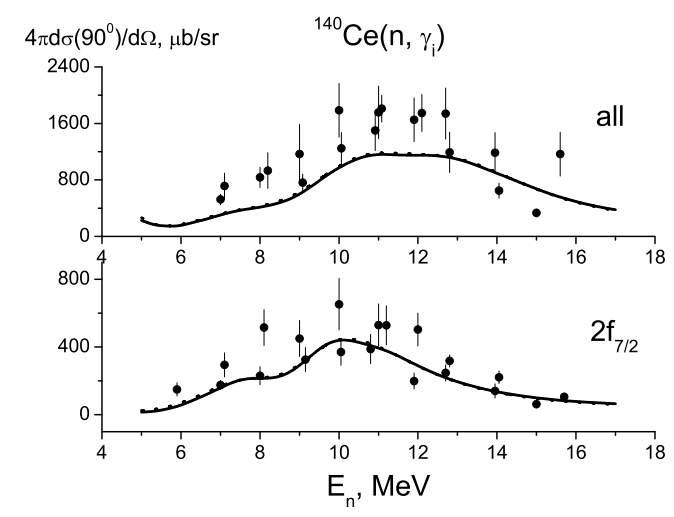

Fig. 5. The calculated partial differential cross sections of the $(n, \gamma)$-reaction for ${ }^{140} \mathrm{Ce}$. The experimental data are taken from Ref. [11].

comparing with the corresponding experimental data. Also the calculated anisotropy parameter $a_{2}$ for some partial ${ }^{208} \mathrm{~Pb}(n, \gamma)$-reactions is shown in figure 7 . In the presented calculation results the experimental spectroscopic factors for s. p. states populated in the radiative capture process are taken from Ref. [12]. In the each presented figure here and below the solid and dotted lines correspond to the results obtained with the use of $\bar{A}_{0}(\omega)$ and the $\bar{A}_{0}^{\text {pole }}(\omega)$, respectively.

As it has been above noted, to study the properties of the IVGQR it is reasonable to research, in particular, the asymmetry of the partial differential cross sections of the $(n, \gamma)$-reaction in this energy region. For this reason it needs to introduce one more adjustable parameter $k_{2}^{\prime}$. As the examples the calculated values of this asymmetry

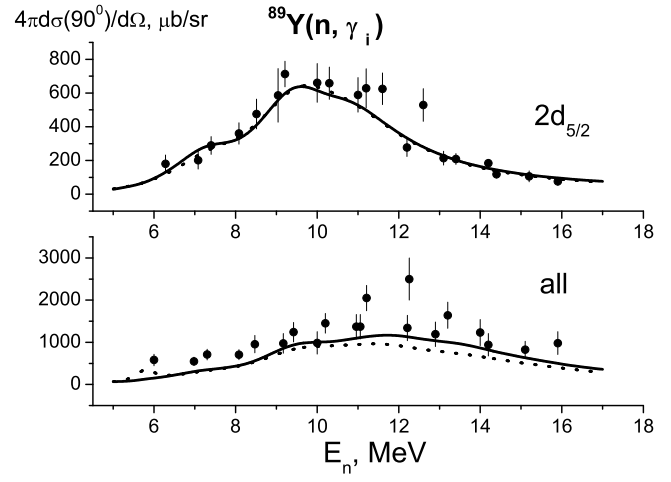

Fig. 6. The calculated partial differential cross sections of the $(n, \gamma)$-reaction for ${ }^{89} \mathrm{Y}$. The experimental data are taken from Ref. [11].

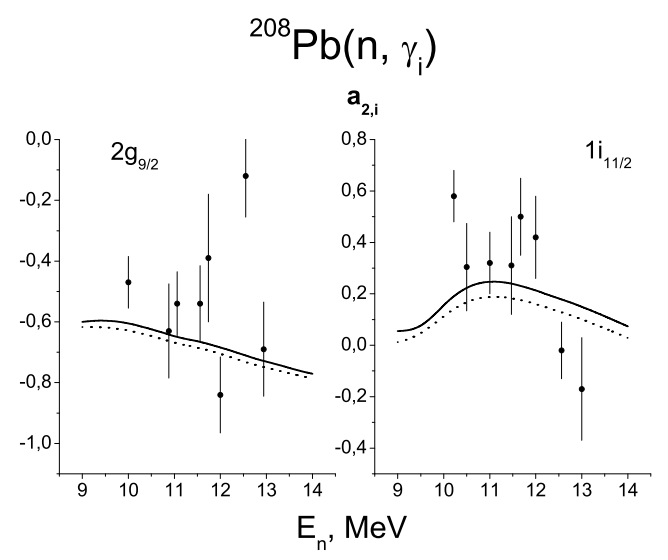

Fig. 7. The calculated anisotropy parameter $a_{2}$ for some partial ${ }^{208} \mathrm{~Pb}(n, \gamma)$-reactions. The experimental data are taken from Ref. [10].

for ${ }^{208} \mathrm{~Pb}$ and ${ }^{209} \mathrm{Bi}$ are shown in figures 8,9 , respectively, in comparison with the corresponding experimental data. These results are obtained, using $k_{2}^{\prime}=0.1$. The IVGQR energy and its other characteristics, evaluated with this value of $k_{2}^{\prime}$, happened to be consistent with the systematic of Ref. [1].

\section{Conclusion}

In this work first implementations of the new semimicroscopic model, the $\mathrm{p}$-h dispersive optical model, are realized. Satisfactory description of the neutron radiative capture accompanied by excitation of the isovector giant dipole and quadrupole resonances in a few medium-heavy mass nuclei is obtained without the use of specific adjustable parameters. Within the mentioned model the verification and the extension (for arbitrary, but high enough, excitation energies) of the semimicroscopic approach to the description of giant resonances (including direct-nucleondecay properties) are achieved. New implementations of the model to describe the simplest photonuclear reactions are in progress. 


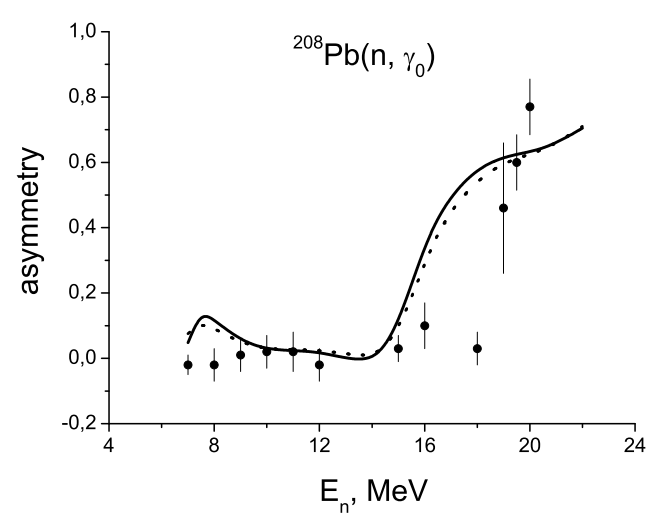

Fig. 8. The calculated asymmetry of the differential cross section of the ${ }^{208} \mathrm{~Pb}\left(n, \gamma_{0}\right)$-reaction in comparison with the experimental data [13].

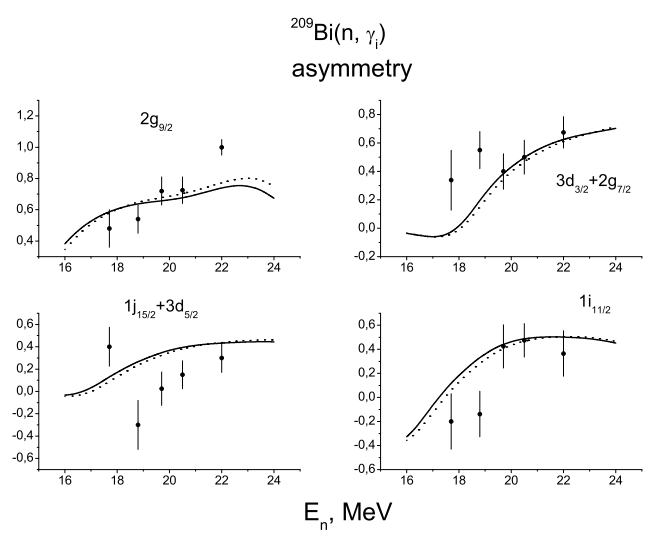

Fig. 9. The calculated asymmetry of partial differential cross sections of the $(n, \gamma)$-reaction for ${ }^{209} \mathrm{Bi}$ in comparison with the experimental data [14].

This work was supported in part by the Russian Fund for Basic Research under grant No. 12-02-01303-a.

\section{References}

1. M.N. Harakeh and A. van der Woude, Giant resonances: Fundamental High-Frequency Modes of $\mathrm{Nu}$ clear Excitations (Oxford, University Press, New York, 2001).

2. M.H. Urin, Nucl. Phys. A 811, 107 (2008)

3. M.H. Urin, Phys. At. Nucl. 74, 1189 (2011)

4. B.A. Tulupov, M.G. Urin, Phys. At. Nucl. 72, 737 (2009)

5. B.A. Tulupov, M.G. Urin, Phys. At. Nucl. 75, 1041 (2012)

6. M.H. Urin, in Proceedings of the Intern. Conference on Nuclear Structure and Related Topics (NSRT12) (Dubna, Russia, July 2-7, 2012)

7. A.B. Migdal, Theory of Finite Fermi Systems and Applications to Atomic Nuclei (Interscience, New York, 1967)

8. S.Yu. Igashov, M.G. Urin, Bull. RAS. Physics. 70, 212 (2008)
9. A. Vessière et al., Nucl. Phys. A 159, 561 (1970); I.N. Boboshin et al, CDFE nuclear data bases, http://depni.npi.msu.su/cdfe.

10. I. Bergqvist, D.M. Drake and D.K. McDaniels, Nucl. Phys. A 191, 641 (1972)

11. I. Bergqvist et al., Nucl. Phys. A 295, 256 (1978)

12. A. Likar and T. Vidmar, Nucl. Phys. A 591, 458 (1995)

13. D.M.Drake, S.Joly et al., Phys. Rev. Lett. 47, 1581 (1981)

14. A. Hakansson, J. Blomgren et al., Nucl. Phys. A 512, 399 (1990) 